\title{
FAST PROXIMAL ALGORITHMS FOR SELF-CONCORDANT FUNCTION MINIMIZATION WITH APPLICATION TO SPARSE GRAPH SELECTION
}

\author{
Anastasios Kyrillidis and Volkan Cevher \\ École Politechnique Fédéral de Lausanne \\ \{anastasios.kyrillidis,volkan.cevher\}@epfl.ch
}

\begin{abstract}
The convex $\ell_{1}$-regularized log det divergence criterion has been shown to produce theoretically consistent graph learning. However, this objective function is challenging since the $\ell_{1}$-regularization is nonsmooth, the log det objective is not globally Lipschitz gradient function, and the problem is high-dimensional. Using the selfconcordant property of the objective, we propose a new adaptive step size selection and present the (F)PS ((F)ast Proximal algorithms for Self-concordant functions) algorithmic framework which has linear convergence and exhibits superior empirical results as compared to state-of-the-art first order methods.
\end{abstract}

Index Terms - Sparse inverse covariance estimation, selfconcordance, step size selection

\section{INTRODUCTION}

Problem setup: Let $\mathcal{X}=\left\{X_{1}, X_{2}, \ldots, X_{n}\right\}$ be a set of variables with joint Gaussian distribution $f\left(X_{1}, X_{2}, \ldots, X_{n}\right) \sim \mathcal{N}(\boldsymbol{\mu}, \boldsymbol{\Sigma})$ where $\boldsymbol{\mu} \in \mathbb{R}^{n}$ is assumed known and $\boldsymbol{\Sigma} \in \mathbb{R}^{n \times n}, \boldsymbol{\Sigma}>0$ denotes the unknown covariance matrix. In this setting, assume we only have access to the underlying model through a set of independent and identically distributed (iid) samples $\left\{\mathbf{x}_{j}\right\}_{j=1}^{p}$ such that $\mathbf{x}_{j} \sim \mathcal{N}(\boldsymbol{\mu}, \boldsymbol{\Sigma}), \forall j$. Given $\left\{\mathbf{x}_{j}\right\}_{j=1}^{p}$, we are interested in inferring any conditional dependencies among $\mathcal{X}$ by estimating $\boldsymbol{\Sigma}^{-1}$. A non-robust estimate of $\boldsymbol{\Sigma}^{-1}$ is through the sample covariance $\widehat{\boldsymbol{\Sigma}}=\frac{1}{p} \sum_{j=1}^{p}\left(\mathbf{x}_{j}-\hat{\boldsymbol{\mu}}\right)\left(\mathbf{x}_{j}-\hat{\boldsymbol{\mu}}\right)^{T}$ where $\hat{\boldsymbol{\mu}}=\frac{1}{p} \sum_{j=1}^{p} \mathbf{x}_{j}$. Unfortunately, in many cases, we cannot afford to acquire adequate samples for accurate $\boldsymbol{\Sigma}^{-1}$ estimation via $\hat{\boldsymbol{\Sigma}}$; for $p \ll n, \widehat{\boldsymbol{\Sigma}}$ is rank-deficient and the use of sophisticated estimation procedures is imperative.

Graphical models interpretation: In undirected graphical models, each variable $X_{i}$ corresponds to a node in a Gaussian Markov random field (GMRF). Moreover, let $E=\left\{(i, j): X_{i}\right.$ 丈 $X_{j} \mid X_{k}$ is observed $\forall k \neq i, j\}$ be the set of edges in the graph. Under this setting, we desire to infer the graph structure given a set of observations. Due to the Gaussianity assumption, $\boldsymbol{\Sigma}_{i j}^{-1}=0 \Leftrightarrow(i, j) \notin E$.

Optimization criteria: [1] shows that the maximum likelihood estimation $\left(\boldsymbol{\Sigma}^{*}\right)^{-1}=\arg \max _{\boldsymbol{\Sigma}^{-1}>0} \prod_{j=1}^{p} f\left(\mathbf{x}_{j}\right)$ is equivalent to:

$$
\boldsymbol{\Theta}^{*}=\underset{\boldsymbol{\Theta}>0}{\operatorname{argmin}}\{-\log \operatorname{det}(\boldsymbol{\Theta})+\operatorname{tr}(\boldsymbol{\Theta} \hat{\boldsymbol{\Sigma}})\},
$$

where $\Theta^{*}=\left(\Sigma^{*}\right)^{-1}$. Based on (1), developments in random matrix theory [2] divulge the poor performance of $\Theta^{*}$ without regularization: the solution to (1) is usually fully dense and no inference

This work was supported in part by the European Commission under Grant MIRG-268398, ERC Future Proof, ARO MURI W911NF0910383, and SNF 200021-132548. VC also would like to acknowledge Rice University for his Faculty Fellowship. about the graph structure is possible. Moreover, when $p \ll n$, the absence of a regularization term leads to non-robust estimates of $\Sigma^{-1}$.

In practice though, parsimonious solutions that adequately explain the data, increase the interpretability of the results even if they lead to worse-valued loss objective values. Using $\ell_{1}$-norm to regularize the objective, (1) can be well-approximated by:

$$
\boldsymbol{\Theta}^{*}=\underset{\boldsymbol{\Theta}>0}{\arg \min }\{F(\boldsymbol{\Theta}):=f(\boldsymbol{\Theta})+g(\boldsymbol{\Theta})\},
$$

where $f(\boldsymbol{\Theta}):=-\log \operatorname{det}(\boldsymbol{\Theta})+\operatorname{tr}(\hat{\boldsymbol{\Sigma}} \boldsymbol{\Theta})$ and $g(\boldsymbol{\Theta}):=\rho\|\operatorname{vec}(\boldsymbol{\Theta})\|_{1}$ with $\rho>0$ that defines the sparsity of the graph selection.

Challenges: Within this context, the main challenges in (2) are:

- High-dimensional problems have become the norm in data analysis; thus, time- and memory-efficient schemes are crucial.

- Apart from its computational challenge, (2) is a non-trivial convex problem: $f(\boldsymbol{\Theta})$ is a strictly convex but not globally Lipschitzcontinuous gradient function; moreover, $g(\boldsymbol{\Theta})$ is a nonsmooth regularizer. Even in simple gradient descent schemes, Lipschitzbased optimal step size calculation becomes infeasible and heuristics lead to slowly convergent, state-of-the-art algorithms [3]. Moreover, (2) is constrained over the set of positive-definite matrices and the choice of regularization parameter $\rho$ is crucial [4].

Prior work: Being a special case of semidefinite programming, (2) can be solved using off-the-shelf interior point approaches [5, 6]. Though, the resulting per iteration complexity for existing interior point methods is $\mathcal{O}\left(n^{6}\right)$ [7]. This has led to the development of multifarious works, which can be roughly categorized into five camps: (i) first-order gradient methods [7, 8, 9], (ii) second order (Newtonbased) gradient methods [10,11], (iii) interior point-based schemes [12], (iv) Lagrangian [13,3] and (iii) greedy approaches [14].

While many of the first-order approaches are slowly convergent and require numerous parameters to be set apriori (reducing their universality), recent developments on second-order methods have resulted in very fast solvers. Though, to achieve this fast performance, these approaches "sacrifice" their universality for faster implementation: one can envision complicated examples (e.g., non-modular regularization) where second-order approaches fail to use their "arsenal" (e.g., greedy heuristics) for computational superiority.

Contributions: Our contributions can be summarized as follows:

- We introduce a new adaptive step size for first-order methods to solve (2), based on the self-concordance property. This technique can be incorporated in mane other minimization problems with the same property. Moreover, this tool can be subsumed in many existing schemes [3] with a wide range of diverse regularization terms, decreasing their time-complexity. 
- To illustrate the substance of the step size selection, we propose the (F)PS ((F)ast Proximal algorithms for Self-concordant functions) framework and show its computational- and memoryefficiency. The resulting schemes have fast convergence and require the minimum number of input parameters.

\section{PRELIMINARIES}

Notation: We reserve lower-case and bold lower-case letters for scalar and vector representation, respectively. Upper-case letters denote matrices. The inner product between matrices $\mathbf{A}, \mathbf{B} \in \mathbb{R}^{m \times n}$ is denoted as $\operatorname{tr}\left(\mathbf{A}^{T} \mathbf{B}\right)$, where $\operatorname{tr}(\cdot)$ is the trace operator. Given a matrix $\mathbf{A} \in \mathbb{R}^{n \times n}$, we reserve $\operatorname{diag}(\mathbf{A}) \in \mathbb{R}^{n \times n}$ to denote the diagonal matrix with entries taken from the diagonal of $\mathbf{A}$.

We reserve $\mathbb{R}_{++}$to denote the set of positive scalars. Let $\mathbb{S}_{++}^{n}$ denote the set of positive definite $n \times n$ matrices. For $p(\mathbf{X}): \mathbb{S}_{++}^{n} \rightarrow$ $\mathbb{R}$, the gradient is denoted as $\nabla p(\mathbf{X})$; for $h(x): \mathbb{R} \rightarrow \mathbb{R}$, we use $h^{\prime}(x), h^{\prime \prime}(x), h^{\prime \prime \prime}(x)$ to denote the first, second and, third derivative.

Definition 1 (Bregman divergence). Let $p: \mathbb{S}_{++}^{n} \rightarrow \mathbb{R} \cup\{+\infty\}$ be a continuously differentiable and strictly convex function. Given $\Theta_{1}, \Theta_{2} \in \mathbb{R}^{n \times n}$, the Bregman divergence $\mathcal{D}_{p}(\cdot \| \cdot)$ is given by:

$$
\mathcal{D}_{p}\left(\boldsymbol{\Theta}_{1} \| \boldsymbol{\Theta}_{2}\right)=p\left(\boldsymbol{\Theta}_{1}\right)-p\left(\boldsymbol{\Theta}_{2}\right)-\operatorname{tr}\left(\nabla p\left(\boldsymbol{\Theta}_{2}\right)\left(\boldsymbol{\Theta}_{1}-\boldsymbol{\Theta}_{2}\right)\right) .
$$

Definition 2 (Convexity bounds in gradient methods). Let $p$ : $\mathbb{S}_{++}^{n} \rightarrow \mathbb{R}$ be a strongly convex function with continuous Lipschitz gradient $\nabla p(\mathbf{X})$ for $\mathbf{X} \in \mathbb{S}_{++}^{n}$. Then, there exist $\mu, L>0$ such that, for any $\boldsymbol{\Theta}_{1}, \Theta_{2} \in \mathbb{S}_{++}^{n}: \frac{\mu}{2} \leqslant \frac{\mathcal{D}_{p}\left(\Theta_{1} \| \Theta_{2}\right)}{\left\|\Theta_{1}-\Theta_{2}\right\|_{F}^{2}} \leqslant \frac{L}{2}$.

Proposition 1 (Step size selection for strongly convex gradient descent schemes). For strongly convex (unconstrained) minimization problems $\min \mathbf{x} q(\mathbf{X})$ where $q: \mathbb{R}^{n \times n} \rightarrow \mathbb{R}, \tau^{*}:=2 /(\mu+L)$ is the optimal step size in the gradient descent scheme $\mathbf{X}_{i+1}=$ $\mathbf{X}_{i}-\tau^{*} \nabla q\left(\mathbf{X}_{i}\right)[15]$.

Definition 3 (Second order expansion of a function). [16] Let $h$ : $\mathbb{R} \rightarrow \mathbb{R}$ be a twice differentiable over an open sphere $\mathcal{S}$. Then, for $x, y \in \mathcal{S}$, there exists an constant $\alpha \in[0,1]$ such that:

$$
h(x+y)=h(x)+h^{\prime}(x) \cdot y+\frac{1}{2} y^{2} \cdot h^{\prime \prime}(x+\alpha y) .
$$

Definition 4 (Self-concordant functions). [17] A convex function $h$ : $\mathbb{R} \rightarrow \mathbb{R}$ is self-concordant if $\left|h^{\prime \prime \prime}(x)\right| \leqslant 2 h^{\prime \prime}(x)^{3 / 2}, \forall x \in \mathbb{R}$. Given two self-concordant functions $h_{1}, h_{2}, h_{1}+h_{2}$ is self-concordant.

Lemma 1 (Upper and lower bounds on second derivatives for self-concordant functions). [17] Let $h: \mathbb{R} \rightarrow \mathbb{R}$ be a strictly convex, self-concordant function. Then, $h^{\prime \prime}(t)$ satisfies:

$$
\frac{h^{\prime \prime}(0)}{\left(1+t \sqrt{h^{\prime \prime}(0)}\right)^{2}} \leqslant h^{\prime \prime}(t) \leqslant \frac{h^{\prime \prime}(0)}{\left(1-t \sqrt{h^{\prime \prime}(0)}\right)^{2}},
$$

where both bounds are valid for $0 \leqslant t<1 / \sqrt{h^{\prime \prime}(0)}$.

\section{GRAPH SELECTION VIA PROXIMAL METHODS}

Given that $F(\boldsymbol{\Theta}):=f(\boldsymbol{\Theta})+g(\boldsymbol{\Theta})$ is strictly convex and provided a putative solution $\Theta_{i} \in \mathbb{S}_{++}^{n}$, an iterative descent scheme follows:

$$
\boldsymbol{\Theta}_{i+1}=\boldsymbol{\Theta}_{i}+\tau_{i}^{*} \boldsymbol{\Delta},
$$

where $\boldsymbol{\Delta} \in \mathbb{R}^{n \times n}$ is a descent direction such that $F\left(\Theta_{i+1}\right)<$ $F\left(\Theta_{i}\right)$ for $\tau_{i}^{*}>0$. To compute $\left\{\boldsymbol{\Delta}, \tau_{i}^{*}\right\}$, we can form the following optimization problem:

$$
\left\{\boldsymbol{\Delta}, \tau_{i}^{*}\right\}=\underset{\boldsymbol{\Delta} \in \mathbb{R}^{n \times n}, \tau>0}{\arg \min }\left\{F\left(\boldsymbol{\Theta}_{i}+\tau \boldsymbol{\Delta}\right): \boldsymbol{\Theta}_{i}+\tau \boldsymbol{\Delta}>0\right\} .
$$

While (4) is the proper way to compute a direction $\Delta$ and a corresponding step size $\tau_{i}^{*}$, in this paper we present an approximation scheme to (4) that introduces the notion of self-concordance in step size selection and performs extremelly well in practice; we reserve the detailed convergence analysis for an extended version.

To this end, the proposed algorithm iteratively computes a putative solution by forming a quadratic surrogate only for $f(\boldsymbol{\Theta})$ at $\boldsymbol{\Theta}_{i} \in$ $\mathbb{S}_{++}^{n}$, i.e., $f(\boldsymbol{\Theta}) \leqslant U\left(\boldsymbol{\Theta}, \boldsymbol{\Theta}_{i}\right):=f\left(\boldsymbol{\Theta}_{i}\right)+\operatorname{tr}\left(\boldsymbol{\Delta} \cdot\left(\boldsymbol{\Theta}-\boldsymbol{\Theta}_{i}\right)\right)+$ $\frac{1}{2 \tau_{i}^{*}}\left\|\boldsymbol{\Theta}-\boldsymbol{\Theta}_{i}\right\|_{F}^{2}$, for a carefully selected $\tau_{i}^{*}>0$ and a direction satisfying $\boldsymbol{\Delta}:=-\nabla f\left(\boldsymbol{\Theta}_{i}\right)$, depending only on $f(\cdot)$, i.e., we ignore the presence of $g(\cdot)$ in $F(\cdot)$. Then, instead of minimizing (2), we iteratively solve the following problem:

$$
\boldsymbol{\Theta}_{i+1}=\underset{\boldsymbol{\Theta}>0}{\arg \min }\left\{U\left(\boldsymbol{\Theta}, \boldsymbol{\Theta}_{i}\right)+g(\boldsymbol{\Theta})\right\},
$$

which can be equivalently stated in proximity operator form [18] as:

$$
\boldsymbol{\Theta}_{i+1}=\underset{\boldsymbol{\Theta}>0}{\arg \min }\left\{\frac{1}{2 \tau_{i}^{*}}\left\|\boldsymbol{\Theta}-\left(\boldsymbol{\Theta}_{i}+\tau_{i}^{*} \boldsymbol{\Delta}\right)\right\|_{F}^{2}+g(\boldsymbol{\Theta})\right\} .
$$

The recursive relation in (6) proposes an optimization recipe : given a step size $\tau_{i}^{*}$, we perform a gradient descent step $\boldsymbol{\Theta}_{i}+\tau_{i}^{*} \boldsymbol{\Delta}$ where $\boldsymbol{\Delta}:=-\nabla f\left(\boldsymbol{\Theta}_{i}\right)$ followed by a soft-thresholding operation $\boldsymbol{\Theta}_{i+1}=$ Soft $\left(\mathbf{X}_{i}, \tau_{i}^{*} \rho\right)$ with threshold $\tau_{i}^{*} \rho$ as the closed-form solution the the proximity operator in (6). Finally, we perform a projection onto the positive definite cone using eigenvalue decomposition.

\section{4. $\tau_{i}^{*}$ SELECTION FOR SELF-CONCORDANT FUNCTIONS}

Given $\boldsymbol{\Delta}:=-\nabla f\left(\boldsymbol{\Theta}_{i}\right)$, we perform a gradient descent step $\mathbf{X}_{i}=$ $\boldsymbol{\Theta}_{i}-\tau_{i}^{*} \nabla f\left(\boldsymbol{\Theta}_{i}\right)$ where $\tau_{i}^{*}>0$ and $\nabla f\left(\boldsymbol{\Theta}_{i}\right):=-\boldsymbol{\Theta}_{i}^{-1}+\hat{\boldsymbol{\Sigma}}$. Since $\tau_{i}^{*}$ is unknown, for clarity let $\mathbf{X}_{i}=\boldsymbol{\Theta}_{i}-\tau \nabla f\left(\boldsymbol{\Theta}_{i}\right)$ where $\tau$ is the unknown variable step size. Then, for $\boldsymbol{\Theta}_{1}:=\mathbf{X}_{i}$ and $\boldsymbol{\Theta}_{2}:=\boldsymbol{\Theta}_{i}$ in Bregman divergence, we define function $\phi(\tau)$ as:

$$
\begin{aligned}
\phi(\tau) & :=\mathcal{D}_{f}\left(\mathbf{X}_{i} \| \boldsymbol{\Theta}_{i}\right)=-\log \operatorname{det}\left(\mathbf{X}_{i}\right)+\log \operatorname{det}\left(\boldsymbol{\Theta}_{i}\right) \\
& +\operatorname{tr}\left(\boldsymbol{\Theta}_{i}^{-1}\left(\mathbf{X}_{i}-\boldsymbol{\Theta}_{i}\right)\right) \\
& =-\log \operatorname{det}\left(\boldsymbol{\Theta}_{i}-\tau \nabla f\left(\boldsymbol{\Theta}_{i}\right)\right)+\log \operatorname{det}\left(\boldsymbol{\Theta}_{i}\right) \\
& -\tau \cdot \operatorname{tr}\left(\boldsymbol{\Theta}_{i}^{-1} \nabla f\left(\boldsymbol{\Theta}_{i}\right)\right) .
\end{aligned}
$$

In (7), we can rewrite the first $\log \operatorname{det}(\cdot)$ term as [17]:

$-\log \operatorname{det}\left(\boldsymbol{\Theta}_{i}-\tau \nabla f\left(\boldsymbol{\Theta}_{i}\right)\right)=-\log \operatorname{det}\left(\boldsymbol{\Theta}_{i}\right)-\sum_{j=1}^{n} \log \left(1-\tau \lambda_{j}\right)$,

where $\lambda_{j}$ are the eigenvalues of $\boldsymbol{\Theta}_{i}^{-1 / 2} \nabla f\left(\boldsymbol{\Theta}_{i}\right) \boldsymbol{\Theta}_{i}^{-1 / 2}$. Then:

$$
\phi(\tau)=-\sum_{j=1}^{n} \log \left(1-\tau \lambda_{j}\right)-\tau \cdot \operatorname{tr}\left(\boldsymbol{\Theta}_{i}^{-1} \nabla f\left(\boldsymbol{\Theta}_{i}\right)\right),
$$

which is a self-concordant function as the superposition of a selfconcordant and a linear (thus self-concordant) function.

Remark 1. In (8), we assume $1-\tau \lambda_{j} \geqslant 0, \forall j$ by the definition of the logarithm function. Subsequently, we show that our step size selection always satisfies these conditions, $\forall j$. 
We observe that (8) is strictly convex as a function of $\tau$. Applying the second order expansion (Definition 3 ) on $\phi(\tau)$, we have:

Lemma 2. The function $\phi(\tau)$ satisfies: $\phi(\tau)=\frac{1}{2} \cdot \tau^{2} \cdot \phi^{\prime \prime}(\hat{\tau})$, for $\hat{\tau} \in[0, \tau]$ and $\phi^{\prime \prime}(\hat{\tau})=\sum_{j=1}^{n} \frac{\lambda_{j}^{2}}{\left(1-\hat{\tau} \lambda_{j}\right)^{2}}$.

Proof. For $y:=\tau, x:=0$ and $\alpha \cdot y:=\hat{\tau}$ in Definition 3, the second order expansion of $\phi(\tau)$ satisfies according to (3):

$$
\phi(\tau)=\phi(0)+\phi^{\prime}(0) \cdot \tau+\frac{1}{2} \cdot \tau^{2} \cdot \phi^{\prime \prime}(\hat{\tau})
$$

It is easy to verify the following: $(i) \phi(0)=0,(i i) \phi^{\prime \prime}(\hat{\tau})=$ $\sum_{j=1}^{n} \frac{\lambda_{j}^{2}}{\left(1-\hat{\tau} \lambda_{j}\right)^{2}}$. Moreover, $\phi^{\prime}(0)=\sum_{j=1}^{n} \lambda_{j}-\operatorname{tr}\left(\boldsymbol{\Theta}_{i}^{-1} \nabla f\left(\boldsymbol{\Theta}_{i}\right)\right)$. But $\sum_{j=1}^{n} \lambda_{j}=\operatorname{tr}\left(\boldsymbol{\Theta}_{i}^{-1} \nabla f\left(\Theta_{i}\right)\right)$. Therefore, $\phi^{\prime}(0)=0$.

Let $\xi(\tau):=\frac{\phi^{\prime \prime}(0)}{\left(1+\tau \sqrt{\phi^{\prime \prime}(0)}\right)^{2}}$. Since $\phi(\cdot)$ is self-concordant and strictly convex, the following inequalities hold true for $\hat{\tau} \in(0, \tau]$ :

$$
\xi(\tau) \leqslant \xi(\hat{\tau}) \leqslant \phi^{\prime \prime}(\hat{\tau}) \leqslant \xi(-\hat{\tau}) \leqslant \xi(-\tau) .
$$

From Lemma 2, $\phi^{\prime \prime}(0)=\sum_{j=1}^{n} \lambda_{j}^{2}$. We know that $\operatorname{tr}\left(\mathbf{A}^{k}\right)=$ $\sum_{j=1}^{n} \xi_{j}^{k}$ for $\mathbf{A} \in \mathbb{R}^{n \times n}$ where $\xi_{j}$ are the eigenvalues of $\mathbf{A}$. Thus, $\phi^{\prime \prime}(0)=\sum_{j=1}^{n} \lambda_{j}^{2}=\operatorname{tr}\left(\left(\boldsymbol{\Theta}_{i}^{-1} \nabla f\left(\boldsymbol{\Theta}_{i}\right)\right)^{2}\right)$.

Given (7), Lemma 2 and $\left\|\mathbf{X}_{i}-\boldsymbol{\Theta}_{i}\right\|_{F}^{2}=\tau^{2}\left\|\nabla f\left(\mathbf{Y}_{i}\right)\right\|_{F}^{2}$ :

$\mathcal{D}_{f}\left(\mathbf{X}_{i} \| \mathbf{\Theta}_{i}\right)=\frac{1}{2} \cdot \tau^{2} \cdot \phi^{\prime \prime}(\hat{\tau}) \Rightarrow \frac{\mathcal{D}_{f}\left(\mathbf{X}_{i} \| \mathbf{\Theta}_{i}\right)}{\left\|\nabla f\left(\mathbf{\Theta}_{i}\right)\right\|_{F}^{2}}=\frac{\phi^{\prime \prime}(\hat{\tau})}{2\left\|\nabla f\left(\mathbf{\Theta}_{i}\right)\right\|_{F}^{2}}$

Combining the above equation with (9), we locally have:

$$
\frac{\tilde{\mu}}{2} \leqslant \frac{\mathcal{D}_{f}\left(\mathbf{X}_{i} \| \boldsymbol{\Theta}_{i}\right)}{\left\|\mathbf{X}_{i}-\boldsymbol{\Theta}_{i}\right\|_{F}^{2}} \leqslant \frac{\widetilde{L}}{2}
$$

where $\widetilde{L}=\frac{\delta}{(1-\tau \sqrt{\delta})^{2} \epsilon}$ and $\tilde{\mu}=\frac{\delta}{(1+\tau \sqrt{\delta})^{2} \epsilon}$ for $\delta:=\phi^{\prime \prime}(0)$ and $\epsilon:=\left\|\nabla f\left(\boldsymbol{\Theta}_{i}\right)\right\|_{F}^{2}$.

By Definition 2, a safe step size selection at the $i$-th iteration satisfies $\tau_{i}^{*}:=\tau=2 /(\widetilde{\mu}+\widetilde{L})$ which leads to the following lemma:

Lemma 3. At the $i$-th iteration, the step size $\tau_{i}^{*}=2 /(\tilde{\mu}+\widetilde{L})$ is determined as $\tau_{i}^{*}=\frac{1}{2}\left(-\frac{1}{\epsilon} \pm \sqrt{\frac{1}{\epsilon^{2}}+\frac{4}{\delta}}\right)$. Moreover, $\tau_{i}^{*}$ is guaranteed to satisfy $0 \leqslant \tau_{i}^{*}<\sqrt{\phi^{\prime \prime}(0)}, \forall i$.

Proof. For $\tau_{i}^{*}:=\tau=2 /(\widetilde{\mu}+\widetilde{L})$ we obtain:

$$
\tau=\frac{2}{\frac{\delta}{(1+\tau \sqrt{\delta}) \epsilon}+\frac{\delta}{(1-\tau \sqrt{\delta}) \epsilon}} \Rightarrow \tau^{2}+\frac{1}{\epsilon} \tau-\frac{1}{\delta}=0
$$

with roots $\tau_{\min , \max }=\frac{1}{2}\left(-\frac{1}{\epsilon} \pm \sqrt{\frac{1}{\epsilon^{2}}+\frac{4}{\delta}}\right)$. To use the upper bound in (9), the solution $\tau$ must satisfy $0 \leqslant \tau<1 / \sqrt{\delta}$. We easily observe that $\tau_{\min } \leqslant 0$. For $\tau_{\max }=\frac{1}{2}\left(-\frac{1}{\epsilon}+\sqrt{\frac{1}{\epsilon^{2}}+\frac{4}{\delta}}\right)$, we have: $\tau_{\max } \geqslant 0$ and $\tau_{\max } \leqslant \frac{1}{2}\left(-\frac{1}{\epsilon}+\sqrt{\frac{1}{\epsilon^{2}}}+\sqrt{\frac{4}{\delta}}\right)=\frac{1}{\sqrt{\delta}}$. since $\frac{1}{\epsilon^{2}}+\frac{4}{\delta}>0$. Thus, $\tau_{i}^{*}:=\tau_{\max }$ such that $\tau_{i}^{*}=2 /(\tilde{\mu}+\widetilde{L})$ and $0 \leqslant \tau_{i}^{*}<\frac{1}{\sqrt{\phi^{\prime \prime}(0)}}$.

Remark 2. An alternative step size selection is computed as the minimum root of $\tau_{i}^{*}=1 / \widetilde{L}$. While this scheme performs well, it does not exploit the strong convexity of the smooth term.

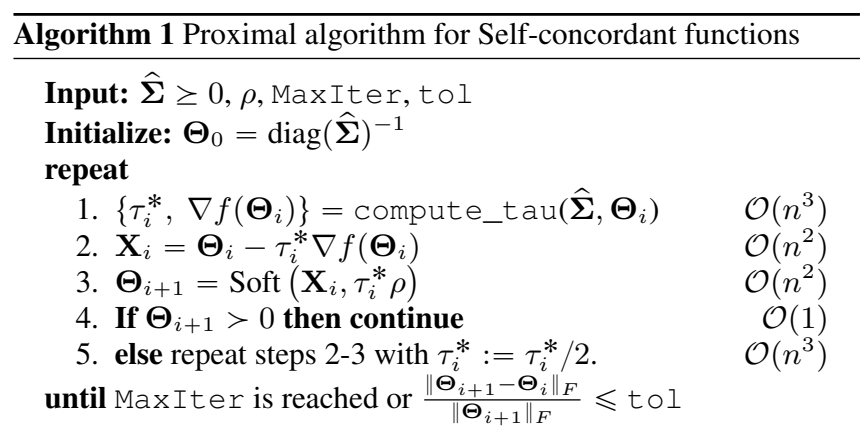

Proposition 2. The step size selection proposed in Lemma 3 satisfies $1-\tau_{i}^{*} \lambda_{j} \geqslant 0, \forall j$ in $(8)$.

Proof. By construction, we observe that $\tau_{i}^{*}<1 / \sqrt{\phi^{\prime \prime}(0)}=$ $\frac{1}{\left(\sum_{j} \lambda_{j}^{2}\right)^{1 / 2}}=1 /\|\boldsymbol{\lambda}\|_{2}$ where $\boldsymbol{\lambda}:=\left[\lambda_{1}, \ldots, \lambda_{n}\right]$. Then,

$$
1-\tau_{i}^{*} \lambda_{j} \begin{cases}\geqslant 0 & \forall j \text { such that } \lambda_{j} \leqslant 0 \text { since } \tau_{i}^{*} \geqslant 0 \\ \geqslant 0 & \forall j \text { such that } \lambda_{j}>0 \text { since } \\ & 1-\tau_{i}^{*} \lambda_{j} \geqslant 1-\frac{\lambda_{j}}{\|\boldsymbol{\lambda}\|_{2}} \geqslant 1-\frac{\|\boldsymbol{\lambda}\|_{\infty}}{\|\boldsymbol{\lambda}\|_{2}} \geqslant 0 .\end{cases}
$$

\section{BASIC PROXIMAL ALGORITHM}

Algorithm 1 shows the Proximal algorithm for Self-concordant functions (PS) in detail. The per iteration complexity is $\mathcal{O}\left(n^{3}\right)$. The step size selection is dominated by the calculation of the gradient $\nabla f\left(\boldsymbol{\Theta}_{i}\right)=-\boldsymbol{\Theta}_{i}^{-1}+\hat{\boldsymbol{\Sigma}}$; an efficient way to compute $\boldsymbol{\Theta}_{i}^{-1}$ is through Cholesky factorization with $\mathcal{O}\left(n^{3}\right)$ complexity. Given $\nabla f\left(\boldsymbol{\Theta}_{i}\right)$ and $\boldsymbol{\Theta}_{i}^{-1}$, the time-complexity for $\delta:=\operatorname{tr}\left(\left(\boldsymbol{\Theta}_{i}^{-1} \nabla f\left(\boldsymbol{\Theta}_{i}\right)\right)^{2}\right)$ and $\epsilon:=$ $\left\|\nabla f\left(\boldsymbol{\Theta}_{i}\right)\right\|_{F}^{2}$ is $\mathcal{O}\left(n^{2}\right)$ while for the quadratic form root-finding step we need $\mathcal{O}(1)$ operations. The soft-thresholding operation requires $\mathcal{O}\left(n^{2}\right)$ complexity.

According to (6), we require $\boldsymbol{\Theta}_{i}>0, \forall i$. The best projection of an arbitrary matrix onto the set of positive definite $n \times n$ matrices requires an eigenvalue decomposition with $O\left(n^{3}\right)$ complexity; a prohibitive time-complexity that does not scale well for many applications. In practice though, the projection onto $\mathbb{S}_{++}^{n}$ can be avoided with a backtrack line search over $\tau_{i}^{*}$. After soft-thresholding, we can check $\boldsymbol{\Theta}_{i+1}>0$ via its Cholesky factorization. In case $\boldsymbol{\Theta}_{i+1} \ngtr 0$, we decrease the step size $\tau_{i}^{*}:=\tau_{i}^{*} / 2$ and repeat steps 2 and 3 with complexity $\mathcal{O}\left(n^{2}\right)$. Otherwise, we can reuse the Cholesky factorization of $\boldsymbol{\Theta}_{i+1}$ to compute $\boldsymbol{\Theta}_{i+1}^{-1}$ and $\nabla f\left(\boldsymbol{\Theta}_{i+1}\right)$ in the next iteration. In practice though, we rarely need this additional operation.

\section{FAST PROXIMAL ALGORITHM}

To gain momentum in convergence, we can use memory in estimates as proposed by Nesterov for strongly convex functions [15]; the same acceleration technique has been integrated in other convex approaches and problems such as [11, 19]. Moreover, to overcome the oscillatory behaviour in the trace of the objective value due to the momentum update, we can use adaptive "restart" techniques; c.f. [20]. Algorithm 2 summarizes the FPS scheme; the main difference with Algorithm 1 is that, at each iteration, we no longer operate on the previous estimate $\boldsymbol{\Theta}_{i-1}$ but rather on $\mathbf{Y}_{i}$ which simulates an 


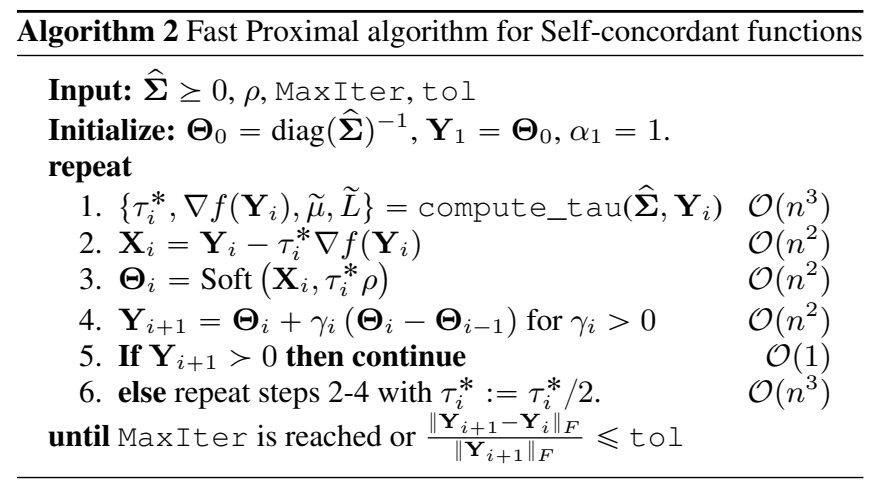

additional (rough) gradient descent step using the previous two estimates $\boldsymbol{\Theta}_{i}$ and $\boldsymbol{\Theta}_{i-1}$. To compute $\nabla f\left(\mathbf{Y}_{i}\right)$ at each iteration, $\mathbf{Y}_{i}$ 's shall satisfy the positive definiteness constraint.

We suggest two schemes for $\gamma_{i}$ [15]: (A): $\gamma_{i}=\left(\frac{\alpha_{i}-1}{\alpha_{i+1}}\right)$ where $\alpha_{i+1}=\frac{1+\sqrt{1+4 \alpha_{i}^{2}}}{2}$ and $\alpha_{1}=1$ and, (B): $\gamma_{i}=\frac{1-\sqrt{\tilde{\mu} \cdot \tau_{i}^{*}}}{1+\sqrt{\tilde{\mu} \cdot \tau_{i}^{*}}}$. We identified that both strategies perform well in practice where scheme (A) is more stable when $\widehat{\boldsymbol{\Sigma}}$ is rank-deficient (non-strictly convex case).

Since we operate on $\mathbf{Y}_{i}$, we have to guarantee the positive definiteness of both $\boldsymbol{\Theta}_{i}$ and $\mathbf{Y}_{i}$ per iteration, leading to an additional Cholesky factorization calculation per iteration. A key lemma for an effcient implementation of Algorithm 2 is the following:

Lemma 4. Given $\boldsymbol{\Theta}_{0}>0, \mathbf{Y}_{i+1}>0$ implies $\boldsymbol{\Theta}_{i}>0, \forall i$.

Proof. If $\mathbf{Y}_{i+1}>0$, then: $\boldsymbol{\Theta}_{i}+\gamma_{i}\left(\boldsymbol{\Theta}_{i}-\boldsymbol{\Theta}_{i-1}\right)>0 \Rightarrow$ $\boldsymbol{\Theta}_{i}\left(1+\gamma_{i}\right)>\gamma_{i} \boldsymbol{\Theta}_{i-1} \Rightarrow \boldsymbol{\Theta}_{i}>\beta_{i} \boldsymbol{\Theta}_{i-1}$, where $\beta_{i}:=\frac{\gamma_{i}}{1+\gamma_{i}}>$ $0, \forall i$. Unfolding the recursion, we have:

$$
\boldsymbol{\Theta}_{i}>\underbrace{\left(\min \left\{\beta_{i}, \beta_{i-1}, \ldots, \beta_{1}\right\}\right)^{i-1}}_{>0} \boldsymbol{\Theta}_{0}>0, \forall i,
$$

By Lemma 4, we can check the positive definiteness of $\boldsymbol{\Theta}_{i}$ through the Cholesky factorization of $\mathbf{Y}_{i+1}$.

\section{EXPERIMENTS}

Experimental configuration: we synthetically generate sparse inverse covariance matrices $\boldsymbol{\Sigma}^{-1}$, according to the simple model:

$$
\boldsymbol{\Sigma}^{-1}=\mathbb{I}+\boldsymbol{\Omega} \text {, such that } \boldsymbol{\Sigma}^{-1}>0 \text { and }\left\|\boldsymbol{\Sigma}^{-1}\right\|_{0}=\kappa,
$$

where $\boldsymbol{\Omega} \in \mathbb{R}^{n \times n}$ contains random iid off-diagonal entries $\sim$ $\mathcal{N}(0,1)$. Given $\boldsymbol{\Sigma}^{-1}$, we draw $\left\{\mathbf{x}_{j}\right\}_{j=1}^{p} \sim \mathcal{N}(\mathbf{0}, \boldsymbol{\Sigma})$ and calculate $\widehat{\boldsymbol{\Sigma}}$. Given the above, we consider two test settings:

(i) $n=1000, p=n / 2$ and, $\kappa=2 \cdot 10^{-3} \cdot n^{2}$. To observe interpretable results, we set $\rho=5 \cdot 10^{-2}$.

(ii) $n=3000, p=5 n$ and, $\kappa=10^{-3} \cdot n^{2}$. To observe interpretable results, we set $\rho=4 \cdot 10^{-2}$.

Linear convergence: We empirically illustrate the convergence rate of the proposed schemes towrads a high-accuracy solution $\Theta^{*}$ of (2); we retain a convergence analysis for an extended version. Let $n=700, p=5 n, \rho=2 \cdot 10^{-2}, \kappa=0.01 n^{2}$. Figure 1 depicts the linear convergence rate of the proposed schemes and their variants; FPSa uses an adaptive restart scheme [20]. In practice, we observe that the choice of $\rho$ heavily affects the condition number of the problem and thus the convergence rate of first-order schemes.

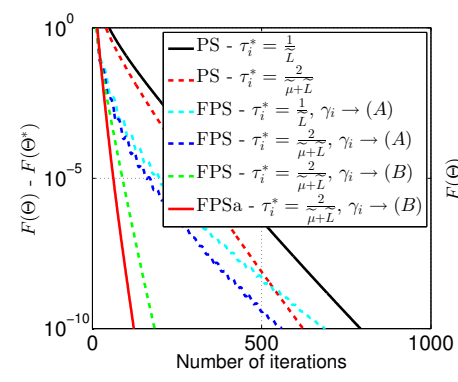

Fig. 1: Convergence rates

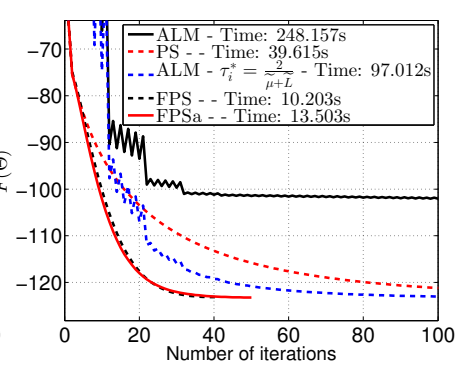

Fig. 2: Comparison plot

\begin{tabular}{c|c|c|c|c} 
Setting $(i)$ & ALM & PS & FPS & FPSa \\
\hline \hline$\left\|\boldsymbol{\Theta}^{*}-\boldsymbol{\Sigma}^{-1}\right\|_{F}$ \\
\hline $\boldsymbol{\Sigma}^{-1} \|_{F}$ & 0.44 & 0.414 & $\mathbf{0 . 4 1 3}$ & $\mathbf{0 . 4 1 3}$ \\
Correct & 1705 & $\mathbf{1 8 9 3}$ & $\mathbf{1 8 9 3}$ & $\mathbf{1 8 9 3}$ \\
Missed & 291 & $\mathbf{1 0 3}$ & $\mathbf{1 0 3}$ & $\mathbf{1 0 3}$ \\
Extra & 365 & 232 & $\mathbf{2 2 8}$ & $\mathbf{2 2 8}$ \\
Iterations & 400 & 379 & 129 & $\mathbf{1 1 4}$ \\
\#Inversions & 400 & 379 & 129 & $\mathbf{1 1 4}$ \\
\hline Setting $(i i)$ & ALM & PS & FPS & FPSa \\
\hline \hline$\left\|\boldsymbol{\Theta}^{*}-\boldsymbol{\Sigma}^{-1}\right\|_{F}$ & - & 0.444 & $\mathbf{0 . 4 3}$ & $\mathbf{0 . 4 3}$ \\
$\left\|\boldsymbol{\Sigma}^{-1}\right\|_{F}$ & - & 8710 & $\mathbf{8 7 2 5}$ & 8724 \\
Correct & - & 290 & $\mathbf{2 7 5}$ & 276 \\
Missed & - & $\mathbf{4}$ & $\mathbf{4}$ & $\mathbf{4}$ \\
Extra & - & 300 & 100 & $\mathbf{9 2}$ \\
Iterations & - & - & $\mathbf{9 2}$ \\
\#Inversions & - & 300 & 100 & $\mathbf{9 2}$ \\
\hline
\end{tabular}

Table 1: "Correct", "Missed" and "Extra" stand for the edges correctly identified, missed or added in the true graph, respectively. MaxIter $=400$ and tol. $=10^{-8}$. “-” depicts no results due to time overhead.

List of algorithms: We compare our scheme against ALM [3], current state-of-the-art first-order gradient method to illustrate the effect of the step size selection. All codes are exclusively written in MATLAB.

Convergence comparison: Figure 2 summarizes the convergence performance of the aforementioned schemes. We simulate test setting $(i)$. Here, "ALM $-\tau_{i}^{*}=\frac{2}{\widetilde{\mu}+\widetilde{L}}$ ", corresponds to ALM [3] using $\tau_{i}^{*}$ in both steps of the algorithm, thus illustrating the universality of our step size selection. All algorithms use $\tau_{i}^{*}=\frac{2}{\widetilde{\mu}+\widetilde{L}}$ and $\gamma_{i} \rightarrow$ (B).

Sparsity pattern recovery performance: For each test setting, we record the median values over 50 Monte-Carlo realizations. Table 1 summarizes the results.

\section{CONCLUSIONS}

Many state-of-the-art gradient approaches for sparse inverse covariance estimation in GMRFs use heuristics to compute a step size which introduce additional "computational losses" due to matrix inversion recalculations or slow convergence. In this work, we present a first-order proximal method which, at its core, utilizes a novel adaptive step size selection procedure based on the self-concordance property of the objective value. Numerical results indicate that our methods overcome state-of-the-art first order methods. Moreover, our framework extends straightforwardly to many convex regularizers; following a simplistic avenue to solve the problem is valuable for the universal application of the algorithm to diverse problems. 


\section{REFERENCES}

[1] J. Dahl, L. Vandenberghe, and V. Roychowdhury. Covariance selection for nonchordal graphs via chordal embedding. Optimization Methods \& Software, 23(4):501-520, 2008.

[2] I.M. Johnstone. On the distribution of the largest eigenvalue in principal components analysis.(english. Ann. Statist, 29(2):295-327, 2001.

[3] K. Scheinberg, S. Ma, and D. Goldfarb. Sparse inverse covariance selection via alternating linearization methods. arXiv preprint arXiv:1011.0097, 2010.

[4] N. Meinshausen and P. Bühlmann. Stability selection. Journal of the Royal Statistical Society: Series B (Statistical Methodology), 72(4):417-473, 2010.

[5] K.C. Toh, M.J. Todd, and R.H. Tütüncü. Sdpt3a matlab software package for semidefinite programming, version 1.3. Optimization Methods and Software, 11(1-4):545-581, 1999.

[6] J.F. Sturm. Using sedumi 1.02, a matlab toolbox for optimization over symmetric cones. Optimization methods and software, 11(1-4):625-653, 1999.

[7] O. Banerjee, L. El Ghaoui, and A. d'Aspremont. Model selection through sparse maximum likelihood estimation for multivariate gaussian or binary data. The Journal of Machine Learning Research, 9:485-516, 2008.

[8] J. Friedman, T. Hastie, and R. Tibshirani. Sparse inverse covariance estimation with the graphical lasso. Biostatistics, 9(3):432-441, 2008.

[9] J. Duchi, S. Gould, and D. Koller. Projected subgradient methods for learning sparse gaussians. arXiv preprint arXiv:1206.3249, 2012.

[10] C.J. Hsieh, M.A. Sustik, I.S. Dhillon, and P. Ravikumar. Sparse inverse covariance matrix estimation using quadratic approximation. Advances in Neural Information Processing Systems (NIPS), 24, 2011.

[11] P.A. Olsen, F. Oztoprak, J. Nocedal, and S.J. Rennie. Newtonlike methods for sparse inverse covariance estimation. Optimization Online, 2012.

[12] L. Li and K.C. Toh. An inexact interior point method for 1 1-regularized sparse covariance selection. Mathematical Programming Computation, 2(3):291-315, 2010.

[13] X. Yuan. Alternating direction methods for sparse covariance selection. preprint, 2009.

[14] K. Scheinberg and I. Rish. Sinco-a greedy coordinate ascent method for sparse inverse covariance selection problem. preprint, 2009.

[15] Y. Nesterov. Introductory lectures on convex optimization. Kluwer Academic Publishers, 1996.

[16] D. Bertsekas. Nonlinear programming. Athena Scientific, 1995.

[17] S. P. Boyd and L. Vandenberghe. Convex Optimization. Cambridge University Press, 2004.

[18] P.L. Combettes and J.C. Pesquet. Proximal splitting methods in signal processing. Fixed-Point Algorithms for Inverse Problems in Science and Engineering, pages 185-212, 2011.

[19] A. Beck and M. Teboulle. A fast iterative shrinkagethresholding algorithm for linear inverse problems. SIAM Journal on Imaging Sciences, 2(1):183-202, 2009.
[20] B. O'Donoghue and E. Candes. Adaptive restart for accelerated gradient schemes. arXiv preprint arXiv:1204.3982, 2012. 